\title{
Impact of Historical Chinese astronomical records on high-energy sources in our Galaxy
}

\author{
Zhenru Wang \\ Department of Astronomy, Nanjing University, Nanjing, 210093, China \\ email: zrwang@nju.edu.cn
}

\begin{abstract}
Owing to the rapid progress of space sciences and the high-energy astrophysics in the past 2-3 decades, historical supernova records have made important contributions to modern astrophysics and will continue to do so in the future. The main topics here are the earliest supernova observed by human beings and the AD393 guest star as well as our new concept of "Po stars"
\end{abstract}

Keywords. Supernova, Historical Supernova; ISM, Supernova Remnant.

\section{Introduction}

Before renaissance, most historical SN (supernova) records are from oriental countries, mainly from China. Biot (1846) and Houmbolds (1850) were the pioneer western astronomers who firstly introduced historical Chinese astronomical records to the West. Since the 20th century more and more astronomers have worked on this field.

Historical SN records are very important because they include the historical clues to the origin and evolution of SNR (supernova remnant) and NS (neutron star) and can provide new information to the active fields of astrophysics and physics. The best example is the famous Tian-Guan guest star with its remnant Crab Nebula (Wang (1987a)). The main topics here are the earliest SN observed and recorded by human beings and AD 393 guest star as well as the new concept of the "Po stars".

\section{The Great New Star}

Which SN is the earliest SN observed and recorded by human beings? My answer is the Great New Star that occurred in the 14th century BC, observed by ancient Chinese and recorded in an oracle bone (see Fig. 1 Left) with the following inscription: "On the 7th day, a Ji-Si night, a Great New Star appeared side by side with the Antares ( $\alpha$ Sco)."From 2nd part of 9th chapter of Yin-Xu-Shu-Qi-Hou-Bian. Antares was an important star to the agriculture and calendar at the ancient time in China.

The Great New Star was considered as a SN by Dong (1945), Xi (1955), Needham (1959) and Xi \& Bo (1965). But they did not consider what its remnant was. In 1987, I proposed that the remnant of the Great New Star be the contemporary $\gamma$-ray source $2 \mathrm{CG}$ $353+16$ discovered by the European $\gamma$-ray satellite COSB. And 2CG 353+16 had been identified as the dark molecular cloud near $\rho$ Oph (Mayer-Hasselwander et al. 1980), but it cannot offer enough $\gamma$-rays to explain the observed $\gamma$-ray flux (Black \& Fazio 1973; Hiller 1984). A good idea is that a SN - the Great New Star - had explored inside there. It can resolve the above difficulty and so 2 CG $353+16$ as the compact remnant (Wang 1987b; Wang 1987c) and diffuse remnant (Xu Wang \& Qu 1992) of the Great New Star are still there. Narike (1999) more exactly determined the age of the Great New Star by use of the 5 lunar eclipses in the reign period of King Wu-ding. 
After searching for the oracle bones, we can find more pieces of them probably related to the Great New Star as shown in Fig. 1 Middle \& Fig. 1 Right a, b, c (taken from Guo 1980; Yao 1988). Combined Fig. 1 Left and Fig. 1 Right, the Great New Star was visible at least 40 days.

If we search the 3rd EGRET Catalogue (Hartman et al. 1999), the Great New Star seems to correspond to 3EG J1627-2419. And there is a note as '...the scale of the variations is much smaller than the pixel size in the EGRET maps. This may lead to apparent sources.' It seems to be helpful for further identification in the future.

\section{AD393 - RX J1713.7-3946 (G347.3-0.5)}

SNR RX J1713.7-3946 was discovered by the ROSAT All Sky Survey (Pfeffermann \& Aschenbach 1996). We suggested it to be the remnant of AD393 guest star (Wang et al. 1997) as the principal basis for four-dimension identification (Wang et al. 1986). The historical record of AD393 guest star is in Fig. 1 (see Wang et al. 1997) and translated as follows : "A guest star appeared within the asterism Wei in the 2nd lunar month and disappeared in the 9th lunar month in the year of AD393 (the 18th year of the Tai-Yuan reign period)".

Both RX J1713.7-3946 and AD393 guest star are in good positional coincidence. The morphology of RX J1713.7-3946 from ROSAT observation seems to be central-ringbrighten expected to have originated from a SN evolving in a stellar wind bubble (Wang et al. 1997). The internal ring is confirmed by XMM-Newton observation (Cassam-Chenie et al. 2004) and the low density feature in the center of the SNR for the stellar wind bubble is obtained as less than $0.02 / \mathrm{cm}^{3}$ by XMM observation(Cassam-Chenaie et al. 2004) and as $0.01 / \mathrm{cm}^{3}$ by $2.6 \mathrm{~mm}$ observation by Fukui et al. (2003).

The distance of RX J1713.7-3946 was in controversy at the beginning around its discovery. The distance of $1 \mathrm{kpc}$ for it was first obtained from ROSAT (Pfeffermann \& Aschenbach 1996). Wang, Qu \& Chen (1997) adopted it as 1kpc. Koyama et al. (1997) also got it as $1 \mathrm{kpc}$ from ASCA observation. But Slane et al. (1999) preferred 6kpc as its distance.

Recently, Fukui et al. (2003) used 4m radio telescope equipped with a sensitive superconducting mixer receiver to observe 1.1 million positions in CO emission. They discovered the interacting molecular gas toward the $\mathrm{TeV} \gamma$-ray peak of the SNR. According to this identification and the measurement for the mean velocity of the non-acceleration component, they determine the distance of the SNR to be $\sim 1 \mathrm{kpc}$ (Moriguchi et al. 2005; Fukui et al. 2003). The column density of HI and CO observation also supports it to be at $1 \mathrm{kpc}$ (Koo et al. 2004). From its XMM observation combined with the CO and HI survey (Cassam-Chenaie et al. 2004), it is pointed out that the CO and HI absorption column densities are in excellent agreement with their XMM measurements in different places of the remnant only if the SNR is at a $1.3 \pm 0.4 \mathrm{kpc}$ and not at $6 \mathrm{kpc}$. Lazendic et al. (2004) suggested that its observed X-ray flux appears problematic to the total energy budget of RX J1713.7-3946 unless the source distance is a factor of 5 smaller than that they preferred before. Hence it seems that a lot of recent observations support it at $1 \mathrm{kpc}$. It will be important for the further investigation of its specific properties.

Now RX J1713.7-3946 is a hot spot in the high-energy astrophysics. Besides it is one of the three shell type SNRs with nonthermal X-ray emission (Koyama et al. 1997; Slane et al. 1997), the TeV $\gamma$-rays were detected from it by CANGAROO (Enomoto et al. 2002) and H.E.S.S. experiment (Aharoniam et al. 2004). It is important whether the TeV $\gamma$-rays originate from the acceleration of protons and the decay of pions (Aharonian \& Atoyan 1999; Enomoto et al. 2002). If it is the case, it would give the first evidence for the 
acceleration of protons in SNRs and then the neutrino emission would be further expected from it (Costantini \& Vissani 2005). It would be very exciting for the development of the origin of cosmic rays and the high-energy astrophysics.

Although there are some different opinion on the emission mechanism of the $\mathrm{TeV} \gamma$ rays by hadronic hypothesis right now, such as Reimer \& Pohl (2002), it can stimulate to study RX J1713.7-3946 in more details both in observation and theoretically. Hopefully GLAST will offer more sensitive and good resolution data of spectra in the band up to Gev and subTeV for clearing up some divergences.

\section{The new concept of "Po Star"}

The bright suspected new star visible for longer than 6 months as a necessary condition (Clark \& Stephenson (1997)) for SNe is certainly applicable only for nearby SNe. We expect distant SNe to be faint and shortly visible. There are many appellations for the ancient suspected new stars, such as guest star, strange star, broom star, and "Po star" etc. The most complicated one is "Po star". "Po star" is a term that was usually used to describe a faint comet or a comet just during its faint phase. So a "Po star" just looked like a very weak comet in visual magnitude, usually without a tail if no other additional notations. Our forbears could discover new faint point source never seen before, but they could not distinguish the nature of them. This is why the concept of a "Po star" came about (Wang Li \& Zhao 2002).

Today a "Po star" can be considered as a faint point source around the visual magnitude of 5. There are about one hundred Po stars recorded from 2320BC to the beginning of 20th century. Xi \& Bo (1965) suggested 7 criteria to distinguish SNe or Novae from them. The main standard is that the object was nonmoving and without tails. 20-30 Po Stars are suggested to be the candidates of SNe or novae (Xi \& Bo 1965; Chen 1986). Based on our new concept of Po star the SGR 1900+14 (Hurley 1999) and the youngest PSR J1846-0258 (Gotthelf et al. 2000) are suggested to be the remnant of BC 4 Po star and AD1523 Po star respectively (Wang Li \& Zhao 2002; Wang 2001).

\section{Acknowledgements}

Thanks to Zhao, Y. and Narike for their helpful discussion on my research, to Li, M. for his help on the manuscript. This work is supported by NNSF of China and the IAU Grant.

\section{References}

Aharonian, F. A. \& Atoyan, A. M. 1999, A\&SA, 351, 330

Aharonian, F. A., et al. (H.E.S.S. Collaboration) 2004, Nature, 432, 75

Biot, E. 1846, Connaissance des Temps Additions, p. 67

Black, J. H. \& Fazio, G. G.1973, ApJ, 185, L7

Cassam-Chenai, G., Decourchelle, A., Ballet, J., Sauvageot, J.-L., \& Dubner, G. 2004, A\&্A, 427,199

Chen, Z. G. 1986, Chinese Astronomical History, Vol. 3 (Taiwen: Ming Wen)

Clark, D. \& Stephenson, F. 1997, The Historical Supernovae (Pergamon, Oxford)

Costantini, M. \& Vissani F. 2005, Astroparticle Physics, 23, 477

Dong, Z. B. 1945, Yin-Li-Pu (A study on the calendar and astronomy of the Shang Dynasty), Sichuan, China

Enomoto, R., et al. (CANGROO Collaboration) 2002, Nature, 416, 823

Fukui, Y., et al. 2003, PASJ, 55, L61

Gotthelf, R., et al. 2000, ApJ, 542, L37 
Guo, M. R. 1980, The Compilation of the Inscription of Tortoise Shells and Bones

Hartman, R. C., et al. 1999, APJS 123, 79-202

Hiller, R. 1984, Gamma Ray Astronomy, Clarendon Press , Oxford

Houmboldt, K. 1850, Kosmos, III, p. 220

Hurley, K. 1999 ApJ, 510, L111

Koo, B., et al. 2004, J. Korean Astron. Soc., 37, 61; IAU Sym. 218, Camilo \& Gaensler eds. 73 Koyama, K. et al. 1997, PASJ, 49, L7

Lazendic, J., et al. 2004, ApJ, 602, 271

Mayer-Hasselwander, H. A., et al. 1980, Proc. Texas Symp. 9th Relativ. Astroph., Ann. NY Acad. Sci., 336, 211

Moriguchi, Y., et al. 2005, in X-Ray and Radio Connections (eds. L. O. Sjouwerman and K. K Dyer)

Narike, T. 1999, AAPPS Bulletin, 9, No. 2, 10

Needham, J. 1959, Science and Civilisation in China, Vol. 3, Cambridge Univ. Press

Pfeffermann, E. \& Aschenbach, B. 1996, in Roentgenstrahlung from the Universe, International Conf. on X-ray Astron. \& Astroph., MPE report 263 (Zimmermann, Trumper \& Yorke ed.), p. 267

Reimer, O. \& Pohl, M. 2002, A\& A, 390, L43

Slane, P., et al. 1997, AAS, 29, 1368

Slane, P., et al. 1999, ApJ, 525, 3

Wang, Z. R., Liu, J. Y., Gorenstein, P., \& Zombeck, M. V. 1986, in Highlights of Astronomy, Vol 7, J. P. Swings ed., p. 583

Wang, Z. R. 1987a, in The Origin and Evolution of Neutron Stars, IAU Symp. 125, Helfand \& Huang ed. (Reidel, Dordrecht), p. 305

Wang, Z. R. 1987b, Science, 235, 1485

Wang, Z. R. 1987c, La Recherche, 18, 1416

Wang, Z. R, Qu, Q. Y., \& Chen, Y. 1997, A\&A, 318, L59

Wang, Z. R. 2001, in New Century of X-ray Astron., ASP Conf. Ser. 251, Inoue \& Kunieda ed., 284

Wang Z. R., Li, Z. Y., \& Zhao, Y. 2002, ApJ, 544, L49

Xi, Z. Z. 1955, Acta Astronomica Sinica, 3, 183

Xi, Z. Z. \& Bo, S. R. 1965, Acta Astronomica Sinica, 13, 1

Xu, J. J., Wang, Z. R., \& Qu, Q. Y. 1992, A\&A, 256.

Yao, X. S. 1988, The compilation of the explanation for facsimile copy of Yin-Xu oracle bones (Beijing-Zhonghua Book Co.)
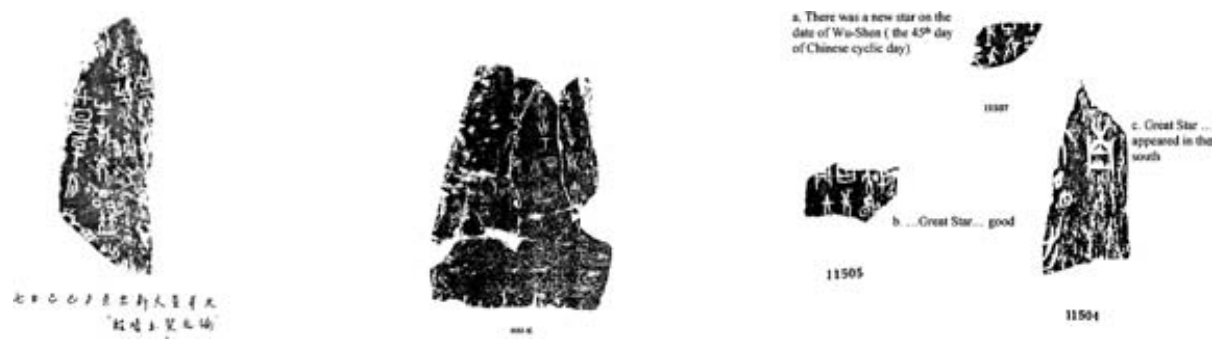

Figure 1. Chinese oracle bone records in the 14th century BC. Left: On the 7th day, a Ji-Si night, a Great New Star appeared side by side with the Antares ( $\alpha$ Sco). Middle: There was a new star on the date of Xin-Wei (the 8th day of Chinese cyclic day) in the Heavenly Stems and Earthly Branches. Right: a. There was a new star on the date of Wu-Shen (the 45th day of Chinese cyclic day) b. ...Great Star...good c. Great Star...appeared in the south. 


\section{Discussion}

MiLEY: From a comparison of multiple reports of the same guest star in ancient records what was the typical positional accuracy of the reports?

WANG: It is hard to give a typical positional accuracy for guest stars. In the ancient records, their positions are usually recorded as near a certain famous star, related to an asterism or a constellation. So the positional accuracy is different for various cases. It depends on the stellar environment nearby the guest star. 